\title{
Classificação geomecânica e análise da estabilidade de taludes em maciço rochoso em trechos das rodovias PE-160 e BR-104
}

Geomechanical classification and stability analysis of road slopes in rock masses in stretches of the PE-160 and BR-104 roadways

Clasificación geomecánica y análisis de estabilidad de taludes en un macizo rocoso en tramos de las carreteras PE-160 y BR-104

Tallys Celso Mineiro

ORCID: https://orcid.org/0000-0001-8044-3503 Universidade Federal de Pernambuco, Brasil E-mail: tallyscelsomineiro@gmail.com

Gabriel Magno Cavalcante Calado ORCID: https://orcid.org/0000-0001-6801-2399 Universidade Federal de Pernambuco, Brasil E-mail: gabrielm2c@hotmail.com

Wagner Leal Guimarães Filho

ORCID: https://orcid.org/0000-0001-5389-4122 Universidade Federal de Pernambuco, Brasil E-mail: wagner.filholg@gmail.com

Ananda Barbosa de Souza

ORCID: https://orcid.org/0000-0002-0562-3608 Universidade Federal de Pernambuco, Brasil E-mail: ananda.b.s19@gmail.com

Shirley Minnell Ferreira de Oliveira ORCID: https://orcid.org/0000-0002-5036-3788 Universidade Federal de Pernambuco, Brasil E-mail: shirley.minnell@ufpe.br

\begin{abstract}
Resumo
Obras de infraestrutura tais como rodovias desenvolvem papel vital no funcionamento e desenvolvimento de uma sociedade; neste caso, sendo essencial para o escoamento têxtil da região. É comum em obras rodoviárias o corte de maciços rochosos resultando em encostas, que fundamentalmente necessitam de estabilidade para o pleno funcinamento das suas vias geratrizes. Este estudo pretende analisar enconstas provenientes da duplicação do trecho da PE-160 e BR-104 entre os municípios de Santa Cruz do Capibaribe e Toritama, com o objetivo de verificar a estabilidade das mesmas utilizando classificações geomecânicas como ferramentas de quantificação e qualificação. Na verificação da estabilidade foi utilizado o RMR (Rock Mass Rating) e SMR (Slope Mass Rating). O RMR se baseia em dados preliminares intrínsecos do maciço rochoso tais como a qualidade da rocha, caracterização das descontinuidades e condições hidrogeológicas; enquanto o SMR adiciona outras duas características ao RMR, que são as atitudes das descontinuidades dos taludes e a sua metodologia de escavação. Com os dados obtidos em campo, calculou-se o RMR dos maciços e este valor foi utilizado para a determinação do SMR que qualifica a condição de estabilidade global do talude. Os resultados obtidos identificaram situações de estabilidade global nos taludes; contudo, observou-se instabilizações localizadas, e para tais condições mínimas, metodologias de melhoramento e proteção foram sugeridas. Logo, a utilização de estudos de estabilidade de taludes como este, podem auxiliar na verificação das condições de segurança da rodovia assegurando-as ou antevendo incidentes e acidentes, e, ajudando em tomadas de decisões.
\end{abstract}

Palavras-chave: Rodovias; Estabilidade de talude; RMR; SMR; Geomecânica.

\begin{abstract}
Infrastructure works such as roads play a vital role in the functioning and development of a society; in this case, being essential for the textile flow of the region. It is common in road works toc ut rock masses resulting in slopes, which fundamentally need stability for the full functioning of its generating routes. This study aims to analyze slopes descendant from the duplication of the PE-160 and BR-104 stretches between the municipalities of Santa Cruz do Capibaribe and Toritama, with the aim of verifying their stability using geomechanical classifications as tools for quantification and qualification. In the verification of stability, RMR (Rock Mass Rating) and SMR (Slope Mass
\end{abstract}


Rating) were used. The RMR is based on preliminary intrinsic data to the rock mass such as rock quality, characterization of discontinuities and hydrogeological conditions; while the SMR adds another two features to RMR, which are the attitudes of the discontinuities of the embankment and its excavation methodology. With the data obtained in the field, the RMR of the massif was calculated and this value was used for the determination of the SMR, that qualifies the global stability condition of the slope. The results obtained identified situations of global stability on the slopes; however, local instabilities were observed and for such minimum conditions, improvement and protection methodologies were suggested. Therefore, the use of slope stability studies like this one can assist in verifying the safety conditions of the roadway, ensuring them or anticipating incidents and accidents, and, helping with decision-making.

Keywords: Roadways; Slope stability; RMR; SMR; Geomechanics.

\section{Resumen}

Las obras de infraestructura, como las carreteras, juegan un papel vital en el funcionamiento y desarrollo de una sociedad; en este caso, es fundamental para el flujo textil en la región. Es común en las obras viales el corte de macizos rocosos dando lugar a taludes, los cuales necesitan fundamentalmente estabilidad para el pleno funcionamiento de sus rutas generadoras. Este estudio tiene como objetivo analizar los taludes derivados de la duplicación del tramo de la PE-160 y BR-104 entre los municipios de Santa Cruz do Capibaribe y Toritama, con el objetivo de verificar su estabilidad utilizando clasificaciones geomecánicas como herramientas de cuantificación y calificación. Para verificar la estabilidad, se utilizaron RMR (Clasificación de masa de roca) y SMR (Clasificación de masa de pendiente). El RMR se basa en datos preliminares intrínsecos del macizo rocoso, como la calidad de la roca, la caracterización de discontinuidades y las condiciones hidrogeológicas; mientras que el SMR agrega otras dos características al RMR, que son las actitudes de las discontinuidades de la pendiente y su metodología de excavación. Con los datos obtenidos en campo se calculó la RMR de los macizos y este valor se utilizó para determinar la SMR que califica la condición de estabilidad global del talud. Los resultados obtenidos identificaron situaciones de estabilidad global en las pistas; sin embargo, se observó inestabilidad localizada, y para tales condiciones mínimas, se sugirieron metodologías de mejora y protección. Por tanto, la utilización de estudios de estabilidad de taludes como este puede ayudar a verificar las condiciones de seguridad de la carretera, asegurándolas o previendo incidencias y accidentes, y ayudando en la toma de decisiones.

Palabras clave: Carreteras; Estabilidad de taludes; RMR; SMR; Geomecánica.

\section{Introdução}

Em obras lineares como rodovias é recorrente, por vezes, que haja a passagem das mesmas por entre alguns maciços rochosos por meio de técnicas de corte (Tonus, 2009). De certa forma, dependendo das características do maciço a ser cortado estes cortes podem proporcionar conformações de taludes rochosos susceptíveis a instabilidade.

Estas condições de instabilidade intrínsecas são concebidas devido as características morfológicas e geológicas do maciço, assim como as conformações fisiográficas onde este se encontra (Tabalipa, 2008). Na existência de interação exterior, a qual pode-se alterar alguns condicionantes estabilizadores e, consequentemente, incidir na ocorrência da alteração do meio físico original é fatídica a aceleração dos processos de desestabilização dos taludes.

Consoante Goodman (1989), instabilizações em taludes rochosos podem acarretar em risco de rupturas e deslizamentos de blocos de rocha, estes fenômenos podem ocorrer de diferentes maneiras, e bastante complexas quando existem múltiplas famílias de descontinuidades em um mesmo maciço. Contudo, estas instabilizações podem ser analisadas de acordo com três tipos de rupturas basicamente, sendo elas planas, em cunha e por tombamento.

Essas possibilidades de ruptura quando analisadas cinematicamente ou por meio de classificações geomecânicas podem ter suas estabilidades avaliadas, e pode-se antever padrões de rompimento de taludes a fim de se mitigar os danos materiais causados.

Logo, a avaliação da estabilidade de certas conformações de taludes rochosos, como os encontrados em obras rodoviárias, podem ser executadas por meio de metodologias empíricas como as classificações geomecânicas RMR - Rock Mass Rating e SMR - Slope Mass Rating, que foram as adotadas, ou até mesmo o Q system (Barton et. al, 1974), o GSI (Hoek, 1983, 1995, Hoek e Brown, 1997) ou o RMi (Palmstrom, 1996, 2000, 2005).

O RMR é um sistema de classificação que leva em consideração fatores geotécnicos físicos do maciço rochoso como 
a qualidade da rocha constituinte (RQD) e algumas características das descontinuidades ocorrentes (Bieniawski, 1989). Enquanto, o SMR configura-se como uma adaptação do RMR, mas que engloba fatores que efetuam uma análise cinemática do talude no maciço rochoso (Romana, 1993).

Assim sendo, em obras rodoviárias, por serem de extrema importância social, as quais ocorram a presença de taludes rochosos é necessário se estudar e compreender a susceptibilidade de movimentações de blocos de rocha ou ruptura de encostas sobre a pista a fim de se evitar eventuais interdições para manutenção destas ocorrências ou até mesmo danos aos usuários de tais rodovias. Logo, o objetivo deste trabalho é classificar geomacanicamente e analisar as condições de estabilidade de taludes rochosos da região de estudo.

\section{Metodologia}

Este presente trabalho tem seu procedimento metodológico fundamentado em três etapas: a que envolve a caracterização e determinação do local a ser analisado, estudo e procedimento técnico de campo para a obtenção dos dados requeridos para utilizar as metodologias preconizadas por Bieniawski (1989) e Romana (2003) e, por fim, a análise da estabilidade dos taludes através do processamento dos dados coletados.

\subsection{Descrição da área de estudo}

A área de estudo escolhida para o presente trabalho situa-se na em trecho da PE-160 e BR-104, no município de Santa Cruz do Capibaribe. A escolha baseou-se, principalmente, pela importância do trecho que dá acesso ao polo comercial de confecções da região que proporciona um fluxo considerável de veículos durante seu funcionamento e contribui para o escoamento das mercadorias têxteis do estado; bem como pela origem e conformação das encostas que, em sua maioria, são originárias devido ao processo de duplicação do trecho de estudo.

A Figura 1 mostra o trecho em questão a ser estudado, que compreende aproximadamente $16,2 \mathrm{~km}$, e a localização dos 8 taludes analisados.

O posicionamento, em coordenadas, dos taludes ao longo do trecho encontra-se na Tabela 1, enfatizando que a coleta destes dados foi feita mediante uso da aplicação Bússola do iPhone.

Tabela 1 - Coordenadas de latitude e longitude dos taludes analisados.

\begin{tabular}{|c|c|c|}
\hline TALUDE & LATITUDE & LONGITUDE \\
\hline Talude 1 & $7^{\circ} 59^{\prime} 37^{\prime \prime} \mathrm{S}$ & $36^{\circ} 5^{\prime} 41^{\prime \prime} \mathrm{O}$ \\
\hline Talude 2 & $7^{\circ} 58^{\prime} 32^{\prime \prime} \mathrm{S}$ & $36^{\circ} 6^{\prime} 18^{\prime \prime} \mathrm{O}$ \\
\hline Talude 3 & $7^{\circ} 58^{\prime} 21^{\prime \prime} \mathrm{S}$ & $36^{\circ} 6^{\prime} 34^{\prime \prime} \mathrm{O}$ \\
\hline Talude 4 & $7^{\circ} 58^{\prime} 21 " \mathrm{~S}$ & $36^{\circ} 6^{\prime} 34^{\prime \prime} \mathrm{O}$ \\
\hline Talude 5 & $7^{\circ} 58^{\prime} 2^{\prime \prime} \mathrm{S}$ & $36^{\circ} 7^{\prime} 8^{\prime \prime} \mathrm{O}$ \\
\hline Talude 6 & $7^{\circ} 58^{\prime} 0^{\prime \prime} \mathrm{S}$ & $36^{\circ} 7^{\prime} 19^{\prime \prime} \mathrm{O}$ \\
\hline Talude 7 & $7^{\circ} 57^{\prime} 32^{\prime \prime} \mathrm{S}$ & $36^{\circ} 11^{\prime} 19^{\prime \prime} \mathrm{O}$ \\
\hline Talude 8 & $7^{\circ} 57^{\prime} 27^{\prime \prime} \mathrm{S}$ & $36^{\circ} 11^{\prime} 24^{\prime \prime} \mathrm{O}$ \\
\hline
\end{tabular}

Fonte: Autor (2021).

Geologicamente, consoante o Serviço Geológico do Brasil - CPRM (2017), a região de estudo se situa-se na porção centro-leste da Província Borborema, a qual é compreendida essencialmente por litotipos pré-cambrianos e pequenos percentuais de rochas cenozóicas.

A geologia é caracterizada principalmente por ortognaisses migmatitícos e sequências supracrustais agrupadas em 
diferentes complexos e numerosos plútons neoproterozóicos. Além disso, no subdomínio Rio Capibaribe a maioria dos afloramentos é evidenciada por ortognaisses de composição granitíca e com metanortositos, caracterizando, portanto, a qualidade e competência dos maciços rochosos da localidade (CPRM, 2017).

Adicionalmente, a região de estudo encontra-se nas proximidades do Lineamento Pernambuco que historicamente se mostra ativa, no entanto, suas atividades de acomodação geológica não atingem grandes magnitudes (CPRM, 2001; Lima Neto, 2013). Todavia, esta característica pode gerar desestabilizações de encostas em maciços rochosos, e que não será considerada neste trabalho.

Figura 1 - Mapa de localização da área de estudo.

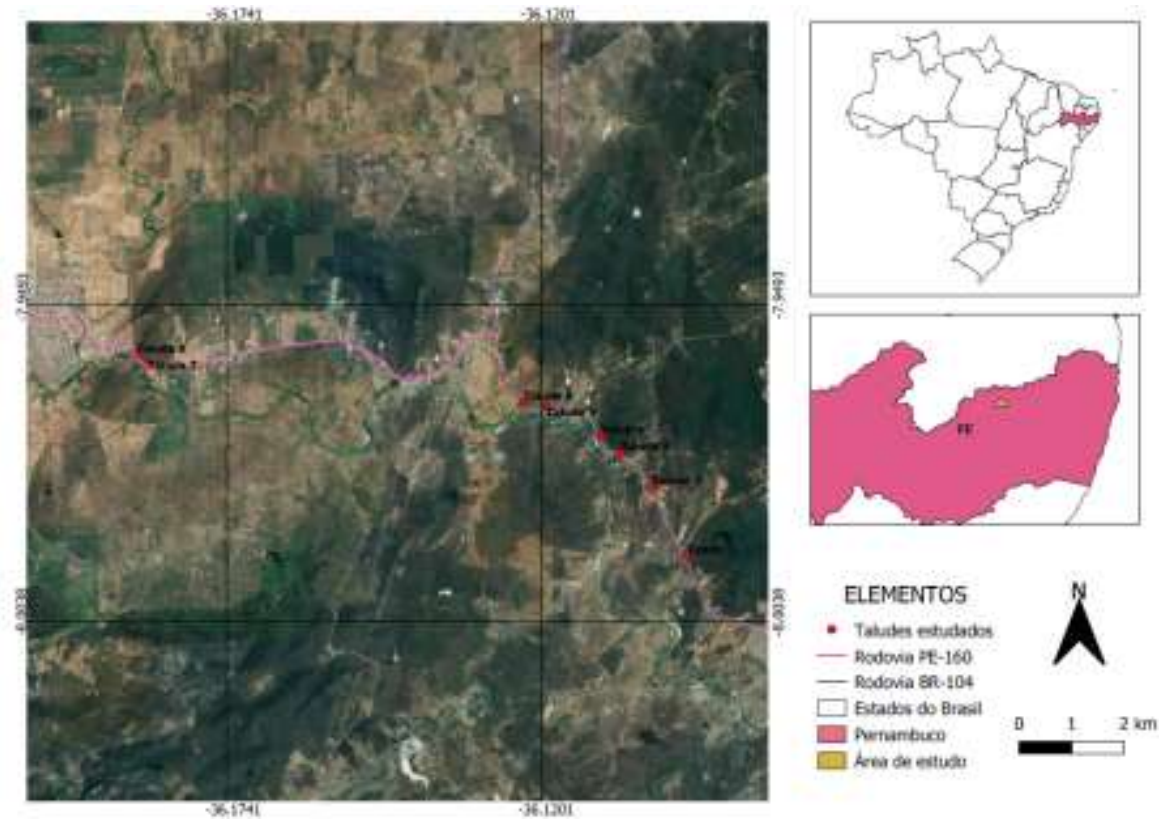

Fonte: Autor (2021).

\subsection{Levantamento geológico-geotécnico}

Para a obtenção dos parâmetros de atitudes dos talude e descontinuidades foi utilizado uma bússola digital, os demais parâmetros relativos as condições geológico-geotécnicas das descontinuidades foram obtidos com o uso de uma fita métrica e uma régua graduada. É importante salientar que certas especificações se obtém a partir de reconhecimento tátil e visual. As descrições obtidas seguiram as normativas da International Society of Rock Mechanics - ISRM (1981) e foram averiguadas consoante as metodologias e terminologias padronizadas por Bieniwaski (1989) e Romana (2003).

Logo, buscou-se verificar as seguintes condições determinísticas para o processamento do índice RMR, que estão descritos na Tabela 2 com suas respectivas faixas de pesos. Estes pesos são somados gerando, por conseguinte, o RMR.

Tabela 2 - Parâmetros necessários para o cálculo do RMR.

\begin{tabular}{cc}
\hline PARÂMETRO & FAIXA DE PESO \\
\hline Resistência da rocha intacta (UCS) & $0-15$ \\
RQD (Rock Quality Designation) & $0-20$ \\
Espaçamento das descontinuidades & $0-20$ \\
Padrão das descontinuidades & $0-30$ \\
Ação da água nas descontinuidades & $0-15$ \\
\hline
\end{tabular}

Fonte: Adaptado Bieniawski (1989). 
No que concerne a resistência da rocha intacta, a mesma pode ser determinada por meio do uso de um esclerômetro de Schmidt ou por meio do uso de um martelo petrográfico, que foi a metodologia adotada no presente trabalho. As normativas da ISRM (1978) esclarecem como determinar uma faixa de resistência para cada amostra e são descritas na Tabela 3.

Tabela 3 - Classificação da resistência da rocha intacta.

\begin{tabular}{|c|c|c|c|}
\hline CLASSE & DESCRIÇÃO & IDENTIFICAÇÃO NO CAMPO & $\begin{array}{c}\text { VALOR APROXIMADO DA } \\
\text { RESISTÊNCIA A CARGA } \\
\text { UNIAXIAL (MPa) } \\
\end{array}$ \\
\hline R0 & Rocha extremamente fraca & Marcada pela unha do polegar & $0,25-1$ \\
\hline $\mathrm{R} 1$ & Rocha muito fraca & $\begin{array}{l}\text { Fragmentada com pancadas firmes } \\
\text { percutidas com a ponta de um martelo de } \\
\text { geólogo, pode ser desagregada com um } \\
\text { canivete }\end{array}$ & $1-5$ \\
\hline $\mathrm{R} 2$ & Rocha fraca & $\begin{array}{l}\text { Pode ser desagregada com um canivete com } \\
\text { dificuldade, marcas superficiais podem ser } \\
\text { feitas com pancadas firmes com a ponta do } \\
\text { martelo de geólogo }\end{array}$ & $5-25$ \\
\hline $\mathrm{R} 3$ & $\begin{array}{l}\text { Rocha medianamente } \\
\text { resistente }\end{array}$ & $\begin{array}{l}\text { Não pode ser desagregada com o canivete, } \\
\text { pode-se obter fragmentos de rocha com uma } \\
\text { pancada de martelo de geólogo }\end{array}$ & $25-50$ \\
\hline $\mathrm{R} 4$ & Rocha resistente & $\begin{array}{l}\text { É requerido mais de uma pancada com o } \\
\text { martelo de geólogo para fraturar a amostra }\end{array}$ & $50-100$ \\
\hline R5 & Rocha muito resistente & $\begin{array}{l}\text { É requerido muitas pancadas com o martelo } \\
\text { de geólogo para fraturar a amostra }\end{array}$ & $100-250$ \\
\hline R6 & $\begin{array}{l}\text { Rocha extremamente } \\
\text { resistente }\end{array}$ & $\begin{array}{l}\text { Apenas pode-se obter lascas da amostra } \\
\text { com o martelo de geólogo }\end{array}$ & $>250$ \\
\hline
\end{tabular}

Fonte: Adaptado ISRM (1978).

Enquanto que para a obtenção do RQD, segundo parâmetro requerido para o RMR e normalmente obtido em ensaio específico de sondagem em rocha, neste caso foi obtido através de correlação. Palmstrom (2005) propôs uma correlação entre o RQD e a densidade volumétrica de descontinuidades por metro cúbico ou densidade volumétrica de juntas (Jv), indicada na Equação 1.

$$
\mathrm{RQD}(\%)=115-3,3(\mathrm{Jv})
$$

Tal correlação assume valor nulo quando a densidade de juntas é maior que 35 e assume valor total para casos em que a densidade seja menor que 4,5.

A densidade volumétrica de juntas, todavia, concerne na quantidade de juntas que interceptam um metro cúbico e é regida pela soma das frações de quantidade de juntas de uma determinada família de descontinuidades pelo seu comprimeito de análise (Palmstrom, 2005). A Equação (2) mostra o processo de cálculo da densidade volumétrica de juntas (Jv).

$$
\mathrm{Jv}=1 / \mathrm{S} 1+1 / \mathrm{S} 2+\ldots+1 / \mathrm{Sn}
$$

Sendo S1, S2 e Sn a média dos espaçamento das juntas. 
Deere (1967) designou uma classificação de acordo com a desempenho dos maciços rochosos a partir do RQD, tal classificação é mostrada na Tabela 4.

Tabela 4 - Classificação do maciço rochoso de acordo com o RQD.

\begin{tabular}{cc}
\hline RQD & QUALIDADE DO MACIÇO \\
\hline $0-25 \%$ & Muito fraco \\
$25-50 \%$ & Fraco \\
$50-75 \%$ & Razoável \\
$75-90 \%$ & Bom \\
$90-100 \%$ & Excelente \\
\hline
\end{tabular}

Fonte: Deere et al. (1967).

Outro parâmetro analisado é o espaçamento entre as descontinuidades que se agrupam em uma determinada família, tal valor associa-se as dimensões dos blocos formadores do maciço, e foi devidamente averiguado consoante a ISRM (1981).

O padrão condicional das descontinuidades é uma descrição conjunta de outros cinco sub-itens que descrevem suas condições geológico-geotécnicas, os quais são: peristência, abertura, rugosidade, preenchimento e grau de alteração.

A persistência corresponde a extensão mensurada de uma descontinuidade em um plano, e caracteriza-se como sendo de grande importância, pois normalmente condiciona o plano de ruptura da encosta rochosa. A ISRM (1981) determinou padrões de classificação para a persistência e estão descritos na Tabela 5.

Tabela 5 - Classificação da persistência de descontinuidades.

\begin{tabular}{cc}
\hline COMPRIMENTO & DESCRIÇÃo \\
\hline$<1$ & Persistência muito baixa \\
$1-3$ & Persistência baixa \\
$3-10$ & Persistência moderada \\
$10-20$ & Persistência alta \\
$>20$ & Persistência muito alta \\
\hline
\end{tabular}

Fonte: Adaptado ISRM (1981).

A abertura consiste na distância entre as faces das superficies limitrófes da junta e pode variar durante sua persistência. Novamente, a ISMR determinou as terminologias de classificação para tal requisito, que são mostradas na Tabela 6.

Tabela 6 - Classificação da abertura de descontinuidades.

\begin{tabular}{cc}
\hline ABERTURA & DESCRIÇÃo \\
\hline$<0,1 \mathrm{~mm}$ & Muito apertada \\
$0,1-0,25 \mathrm{~mm}$ & Apertada \\
$0,25-0,5 \mathrm{~mm}$ & Parcialmente aberta \\
$0,5-2,5 \mathrm{~mm}$ & Aberta \\
$2,5-10 \mathrm{~mm}$ & Moderadamente larga \\
$>10 \mathrm{~mm}$ & Larga \\
$1-10 \mathrm{~cm}$ & Muito larga \\
$10-100 \mathrm{~cm}$ & Extremamente larga \\
$>1 \mathrm{~m}$ & Cavernosa \\
\hline
\end{tabular}

Fonte: Adaptado ISRM (1978). 
A rugosidade é uma propriedade, inerente as juntas, que determina o padrão de irregularização das suas superficies e sua importância consiste em afetar diretamente na resistência ao deslizamento das juntas. Neste trabalho, a metodologia adotada para a classificação da rugosidade foi a utilização da determinação visual por scanline proposta por Barton et al. (1977) através do Joint Roughness Coefficient (JRC), índice tal que determina desde superfície lisa até uma superfície muito rugosa.

O preenchimento concerne no material que integra o espaço entre as paredes adjacentes das descontinuidades. Normalmente originário do intemperismo físico que a rocha foi submetida ou transportado pela água, bem como pode ser inexistente dependendo da mineralogia do maciço. Este conceito com suas propriedades inerentes controla o comportamento da descontinuidade. Neste estudo será utilizada a terminologia descripta na Tabela 7 e proposta por Bieniawski (1989).

Tabela 7 - Classificação do preenchimento de descontinuidades.

\begin{tabular}{c}
\hline DESCRIÇÃO DO PREENCHIMENTO \\
\hline Nenhum \\
Resistente com espessura $<5 \mathrm{~mm}$ \\
Resistente com espessura $>5 \mathrm{~mm}$ \\
Mole com espessura $<5 \mathrm{~mm}$ \\
Mole com espessura $>5 \mathrm{~mm}$ \\
\hline
\end{tabular}

Fonte: Adaptado Bieniawski (1989).

A última caracterização proposta para definir o padrão da descontinuidade é o grau de alteração da rocha que corresponde as condições de decomposição, desagregação e intemperização da mesma. Existem dois tipos de alteração: desintegração mecânica e decomposição química. Na maioria dos casos, as duas resultantes agem concomitantemente; no entanto, dependendo do regime climático uma delas pode ser dominante. A alteração mecânica resulta na abertura de descontinuidades, na formação de novas juntas pelo fraturamento da rocha e a abertura ou clivagem de regiões mineralizadas. Enquanto a decomposição química resulta na descoloração da rocha e conduz a decomposição de silicatos em materiais argilosos (ISRM, 1978). A terminologia da classificação foi determinada consoante a Tabela 8 proposta pela ISRM.

Tabela 8 - Classificação da alteração das descontinuidades na rocha.

\begin{tabular}{cl}
\hline GRAU DE ALTERAÇÃo & \multicolumn{1}{c}{ DESCRIÇão } \\
\hline São ou não-alterada & Não se observa sinais de alteração na rocha, podendo \\
& haver alguma descoloração nas superfícies principais da \\
& descontinuidade. \\
Ligeiramente alterada & Há descoloração indicando alteração da rocha e das \\
& superfícies da descontinuidade. Toda a rocha pode estar \\
& descolorada, podendo a resistência superficial está um \\
& pouco comprometida relativamente ao material intacto. \\
& Menos da metade do material rochoso está decomposto \\
Moderadamente alterada & e/ou desintegrado em solo. \\
& Mais da metade do material rochoso está decomposto e/ou \\
Muito alterada & desintegrado em solo. \\
& Todo o material rochoso está decomposto e/ou \\
Completamente alterada à decomposta & ainda amplamente intacta. \\
\hline
\end{tabular}


Por fim, o parâmetro final analisado é relativo a ação da água na estruturação da descontinuidade. De crucial relevância para a determinação do RMR, pois a mesma é um fator instabilizante e que corresponde a mais simples das caracterizações, uma vez que sua especificação se dar de forma tátil e visual. A classificação adotada e descrita por Bieniawski é mostrada na Tabela 9 .

Tabela 9 - Classificação da ação da água em descontinuidades.

\begin{tabular}{c}
\hline DESCRIÇÃo \\
\hline Completamente seca \\
Ligeiramente úmido \\
Úmido \\
Gotejante \\
Fluente
\end{tabular}

Fonte: Adaptado Bieniawski (1989)

\subsection{Processamento dos dados para cálculo do RMR e SMR}

Obtidos os dados caracterizados, para cada família de descontinuidades e para cada talude, conseguintemente deve-se associar cada parâmetro classificado com seu respectivo peso na sistemática estipulada por Bieniwaski (1989) para calcular o RMR. Na tabela 10 os cinco parâmetros são evidenciados de acordo com sua classificação e sua devida faixa de pesos correspondentes que devem ser somados iterativamente para por fim determinar o índice.

Em casos em que se demanda maior precisão na determinação dos pesos e, portanto, do índice, que é o caso adotado neste estudo, ao invés de utilizar as descrições sucintas estabelecidas para o padrão condicional da descontinuidade utiliza-se o detalhamento mostrado na Tabela 11.

Tabela 10 - Parâmetros e pesos para o cálculo do RMR.

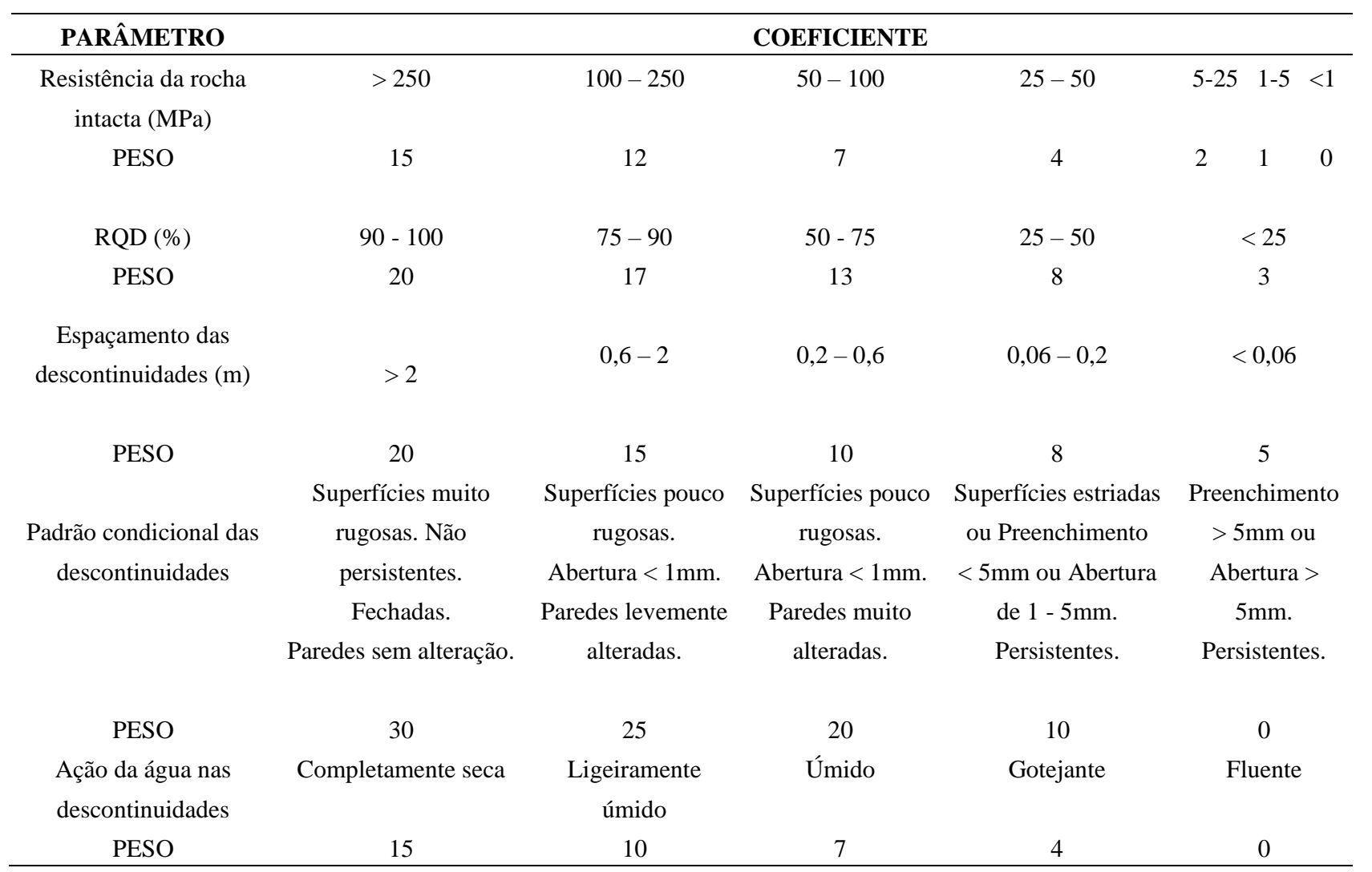

Fonte: Adaptado Bieniawski (1989). 
Tabela 11 - Classificação detalhada do padrão condicional de descontinuidades.

\begin{tabular}{cccccc}
\hline PARÂMETRO & \multicolumn{5}{c}{ DESCRIÇão } \\
\hline Persistência & $<1 \mathrm{~m}$ & $1-3 \mathrm{~m}$ & $3-10 \mathrm{~m}$ & $10-20 \mathrm{~m}$ & $>20 \mathrm{~m}$ \\
PESO & 6 & 4 & 2 & 1 & 0 \\
Abertura & Nenhuma & $<0,1 \mathrm{~mm}$ & $0,1-1 \mathrm{~mm}$ & $1-5 \mathrm{~mm}$ & $>5 \mathrm{~mm}$ \\
PESO & 6 & 5 & 4 & 1 & 0 \\
Rugosidade & Muito rugosa & Rugosa & Pouco rugosa & Suave & Estriadas \\
PESO & 6 & 5 & 3 & 1 & 0 \\
Preenchimento & Nenhum & Duro, $<5 \mathrm{~mm}$ & Duro, $>5 \mathrm{~mm}$ & Mole,$<5 \mathrm{~mm}$ & Mole, $>$ 5mm \\
PESO & 6 & 4 & 2 & 2 & 0 \\
Alteração & Não-alterada & Pouco & Moderada & Altamente & Decomposta \\
PESO & 6 & 5 & 3 & 1 & 0 \\
\hline
\end{tabular}

Fonte: Adaptado Bieniawski (1989).

Bieniawski (1973) qualificou os referidos índices de RMR de acordo com a capacidade autoportante do maciço rochoso, tal qualificação é mostrada na Tabela 12.

Tabela 12 - Classificação da qualidade do maciço de acordo com o RMR.

\begin{tabular}{ccc}
\hline RMR & CLASSIFICAÇÃo & DESCRIÇÃo DO MACIÇO \\
\hline $100-81$ & I & Muito bom \\
$80-61$ & II & Bom \\
$60-41$ & III & Regular \\
$40-21$ & IV & Ruim \\
$<20$ & V & Muito ruim \\
\hline
\end{tabular}

Fonte: Adaptado Bieniawski (1973).

Para a determinação do índice SMR, proposto por Romana (1985) como uma contribuição os sistema de classificação de Bieniawski (1989), utiliza-se como base o valor do RMR adicionado de uma multiplicação de fatores que se definem intrinsicamente a conformidade geométrica de estruturação do talude e suas descontinuidades, bem como a adição de um fator relativo a metodologia de corte do maciço. A Equação 2 mostra a determinação do índice SMR.

$$
\mathrm{SMR}=\mathrm{RMR}+(\mathrm{F} 1 \cdot \mathrm{F} 2 \cdot \mathrm{F} 3)+\mathrm{F} 4
$$

Sendo os fatores de cálculo são determinados pelas Equações 3, 4 e 5, a seguir:

$$
\begin{aligned}
& F 1=[1-\operatorname{sen}(a j-a s)]^{2} \\
& F 2=\operatorname{tg}^{2} b j \\
& F 3=-30+\frac{1}{3} \operatorname{tg}^{-1} \theta
\end{aligned}
$$

Os seguintes parâmetros aj, as, bj e $\theta$ são, respectivamente, nas direções da descontinuidade e do talude, a inclinação da descontinuidade e a diferença entre o mergulho do talude e o mergulho da descontinuidade.

Ao valor F1 pode ser atribuído o valor de 1 quando as direções são paralelas e 0,15 quando a angulação entre as direções é menor que $30^{\circ}$ e a probabilidade de ruptura configura-se como baixa. Enquanto que ao valor de F2 pode ser atribuído o valor de 1 para descontinuidades com inclinações maiores que $45^{\circ}$ e 0,15 para aquelas que cuja inclinação é menor 
que $20^{\circ}$. O fator F4, proveniente do método de escavação, pode assumir os seguintes valores mostrados na Tabela 13.

Tabela 13 - Valores empíricos do fator F4.

\begin{tabular}{cccccc}
\hline $\begin{array}{c}\text { METODOLOGIA } \\
\text { DE ESCAVAÇão }\end{array}$ & $\begin{array}{c}\text { ENCOSTA } \\
\text { NATURAL }\end{array}$ & $\begin{array}{c}\text { DESMONTE DE } \\
\text { PRÉ-CORTE }\end{array}$ & $\begin{array}{c}\text { DETONAÇão } \\
\text { SUAVE }\end{array}$ & $\begin{array}{c}\text { DESMONTE COM EXPLOSIVOS } \\
\text { OU ESCAVAÇÃO MECÂNICA }\end{array}$ & $\begin{array}{c}\text { DESMONTE COM } \\
\text { EXPLOSÃo } \\
\text { INADEQUADA }\end{array}$ \\
\hline PESO & 15 & 10 & 8 & 0 & -8 \\
\hline
\end{tabular}

Fonte: Romana et al. (2003).

Romana (2003) classificou os maciços rochosos de acordo com o SMR e condicinou a estabilidade dos taludes pertencentes a estes maciços, bem como analisou as prováveis rupturas para cada índice condicionante, tais classificações podem ser vistas na Tabela 14.

Tabela 14 - Classificação da qualidade do maciço de acordo com o SMR.

\begin{tabular}{ccccc}
\hline CLASSE & SMR & $\begin{array}{c}\text { DESCRIÇÃO DO } \\
\text { MACIÇO }\end{array}$ & ESTABILIDADE & TIPO DE RUPTURA \\
\hline I & $100-81$ & Muito bom & Completamente estável & - \\
II & $80-61$ & Bom & Estável & Alguns blocos \\
III & $60-41$ & Regular & Parcialmente instável & Rupturas planares ou em cunha \\
IV & $40-21$ & Ruim & Instável & Rupturas planares e em cunha de grandes dimensões \\
V & $<20$ & Muito ruim & Completamente instável & Rupturas de grandes dimensões em planos contínuos \\
\hline
\end{tabular}

Fonte: Adaptado Romana (2003).

Após calculados os índices RMR e SMR, os taludes e seus respectivos maciços rochosos foram classificados de acordo com sua estabilidade, bem como analisadas as prováveis e eventuais rupturas.

\section{Resultados e Discussão}

Esta seção destina-se ao processamento dos dados obtidos em campo a fim de obter as devidas classificações geomecânicas RMR e SMR, respectivamente. Foram analisados 8 taludes, dentre os quais diversas famílias de descontinuidades podem ser encontradas. Neste trabalho analisou-se somente aquelas que favoreciam ou possibilitariam prováveis rupturas planares, em cunha ou por tombamento, as quais foram selecionadas por observação e frequência. Logo, ao todo 23 famílias de descontinuidades foram catalogadas.

\subsection{Caracterização dos taludes e suas respectivas famílias de descontinuidades}

Mediante visita de campo, para cada talude, foram coletados direção e inclinação, direções geológicas e mergulho das respectivas famílias de descontinuidades, e adicionalmente a quantidade de juntas por metro que estavam presente em pontos mais críticos para o cálculo da densidade das mesmas.

A densidade foi obtida pela soma das descontinuidades, uma vez que o volume analisado para o conjunto de famílias foi de $1 \mathrm{~m}^{3}$ e, por conseguinte, obteve-se por correlação o RQD. Os referidos dados podem ser vistos na Tabela 15. 
Tabela 15 - Caracterização dos taludes e suas respectivas famílias.

\begin{tabular}{|c|c|c|c|c|c|c|c|c|}
\hline TALUDE & DIREÇÃO & INCLINAÇÃO & FAMÍLIA & $\begin{array}{c}\text { DIREÇÃO } \\
\text { GEOLÓGICA }\end{array}$ & MERGULHO & $\mathbf{N}_{\text {JUNTAS }} / \mathbf{m}$ & $\mathbf{J}_{\mathbf{V}}$ & RQD \\
\hline \multirow{3}{*}{1} & \multirow{3}{*}{$348^{\circ} \mathrm{N}$} & \multirow{3}{*}{$75^{\circ}$} & F1 & $156^{\circ} \mathrm{SE}$ & $26^{\circ}$ & 5 & \multirow{3}{*}{8} & \multirow{3}{*}{91} \\
\hline & & & F2 & $148^{\circ} \mathrm{SE}$ & $90^{\circ}$ & 1 & & \\
\hline & & & F3 & $59^{\circ} \mathrm{NE}$ & $81^{\circ}$ & 2 & & \\
\hline \multirow{3}{*}{2} & \multirow{3}{*}{$312^{\circ}$ NO } & \multirow{3}{*}{$70^{\circ}$} & F4 & $58^{\circ} \mathrm{NE}$ & $32^{\circ}$ & 3 & \multirow{3}{*}{9} & \multirow{3}{*}{88} \\
\hline & & & F5 & $85^{\circ} \mathrm{L}$ & $-49^{\circ}$ & 3 & & \\
\hline & & & F6 & $320^{\circ} \mathrm{NO}$ & $\mathbf{8 5}^{\circ}$ & 3 & & \\
\hline \multirow[t]{3}{*}{3} & \multirow[t]{2}{*}{$342^{\circ} \mathrm{N}$} & \multirow[t]{2}{*}{$70^{\circ}$} & F7 & $125^{\circ} \mathrm{SE}$ & $-22^{\circ}$ & 9 & \multirow[t]{2}{*}{13} & \multirow[t]{2}{*}{76} \\
\hline & & & F8 & $28^{\circ} \mathrm{NE}$ & $90^{\circ}$ & 4 & & \\
\hline & \multirow{4}{*}{$348^{\circ} \mathrm{N}$} & \multirow{4}{*}{$80^{\circ}$} & F9 & $359^{\circ} \mathrm{N}$ & $90^{\circ}$ & 2 & \multirow{4}{*}{11} & \multirow{4}{*}{82} \\
\hline \multirow[t]{4}{*}{4} & & & F10 & $163^{\circ} \mathrm{S}$ & $90^{\circ}$ & 2 & & \\
\hline & & & F11 & $348^{\circ} \mathrm{N}$ & $26^{\circ}$ & 5 & & \\
\hline & & & F12 & $348^{\circ} \mathrm{N}$ & $-39^{\circ}$ & 2 & & \\
\hline & \multirow{3}{*}{$283^{\circ} \mathrm{O}$} & \multirow{3}{*}{$70^{\circ}$} & F13 & $99^{\circ} \mathrm{L}$ & $-23^{\circ}$ & 4 & \multirow{3}{*}{10} & \multirow{3}{*}{85} \\
\hline \multirow[t]{3}{*}{5} & & & F14 & $219^{\circ} \mathrm{SO}$ & $90^{\circ}$ & 3 & & \\
\hline & & & F15 & $283^{\circ} \mathrm{O}$ & $0^{\circ}$ & 3 & & \\
\hline & \multirow{3}{*}{$295^{\circ} \mathrm{NO}$} & \multirow{3}{*}{$70^{\circ}$} & F16 & $295^{\circ} \mathrm{NO}$ & $20^{\circ}$ & 6 & \multirow{3}{*}{12} & \multirow{3}{*}{79} \\
\hline \multirow[t]{2}{*}{6} & & & F17 & $344^{\circ} \mathrm{N}$ & $85^{\circ}$ & 1 & & \\
\hline & & & F18 & $86^{\circ} \mathrm{L}$ & $70^{\circ}$ & 5 & & \\
\hline \multirow{3}{*}{7} & \multirow{3}{*}{$331^{\circ} \mathrm{NO}$} & \multirow{3}{*}{$75^{\circ}$} & F19 & $273^{\circ} \mathrm{O}$ & $28^{\circ}$ & 2 & & \\
\hline & & & F20 & $123^{\circ} \mathrm{SE}$ & $90^{\circ}$ & 4 & 8 & 91 \\
\hline & & & F21 & $16^{\circ} \mathrm{N}$ & $90^{\circ}$ & 2 & & \\
\hline 8 & $64^{\circ} \mathrm{NE}$ & $70^{\circ}$ & F22 & $155^{\circ} \mathrm{SE}$ & $90^{\circ}$ & 3 & 7 & 94 \\
\hline & & & F23 & $33^{\circ} \mathrm{NE}$ & $45^{\circ}$ & 4 & & \\
\hline
\end{tabular}

Fonte: Autor (2021).

Tendo em vista a heterogeneidade dos maciços e as práticas de corte, os taludes de certa forma não tem uma inclinação definida para sua extensão total, bem como para uma mesma seção pode-se haver irregularidades. Desse modo, a inclinação adotada foi tal que configurasse pela estatística de medida central das inclinações presentes de forma aproximada. Os mergulhos negativos caracterizam descontinuidades que adentram o talude, possibilitando, então, uma ruptura por tombamento.

Em geral, os taludes pela conferência de resistência da rocha intacta ou Ensaio de Compressão Simples (UCS) se mostraram competentes ao ponto de serem necessários vários golpes para a fratura pela metodologia de campo, isto geologicamente, se deve ao fato de tais maciços serem provenientes em sua maioria de ações igneas, uma vez que os mesmos são compostos por granitóides.

No que concerne as descontinuidades, de forma genérica, possuem persitência consideráveis com aberturas que podem variar de $1 \mathrm{~mm}$ à maiores que $5 \mathrm{~mm}$ sem presença considerável de preenchimento e sob condições secas, variando com significância suas alterações e rugosidades.

Os taludes em sua maioria são semelhantes tendo alturas por volta dos 5 metros e extensões de 100 à 200 metros, sendo somente o talude 6 distinto com altura de aproximadamente 20 metros. Pode-se observar na Figura 2 as conformações, vistas em campo, para cada um dos taludes de acordo com sua localização baseado no mapa da Figura 1. 
Research, Society and Development, v. 10, n. 16, e209101623637, 2021

(CC BY 4.0) | ISSN 2525-3409 | DOI: http://dx.doi.org/10.33448/rsd-v10i4.23637

Figura 2 - Conformação dos taludes de acordo com a localização.

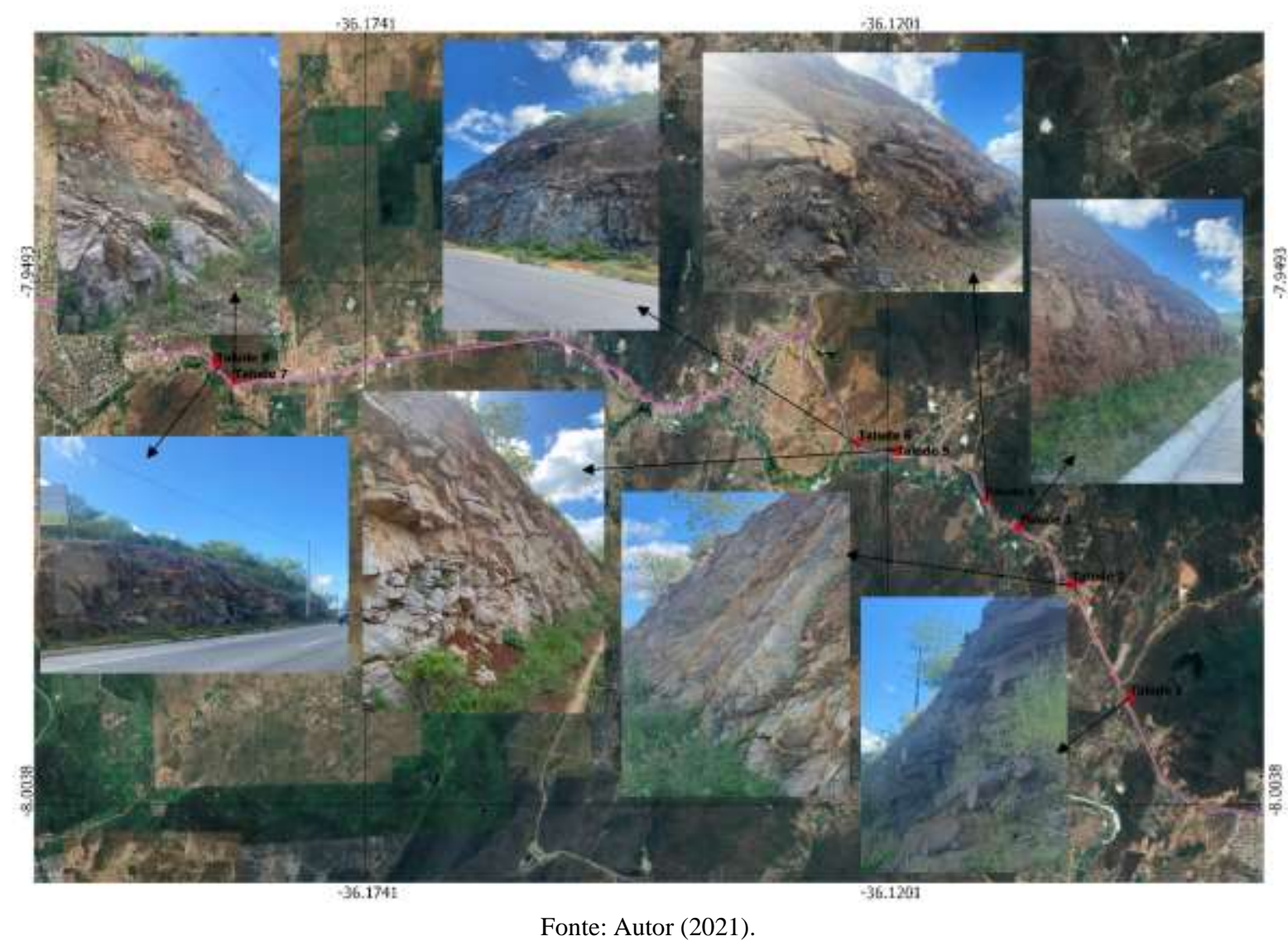

\subsection{Determinação do RMR dos taludes}

Os índices de classificação RMR, foram compilados de acordo com Bieniawski (1973), a partir dos dados coletados em campo, e posteriormente serão base para a determinação do SMR. Este valor corresponde a competência do maciço rochoso e tem amplitude de valores que vai de 0 à 100. A Tabela 16 expõe as condições em que se encontram as descontinuidades e o maciço rochoso de acordo com seus respectivos pesos. 
Tabela 16 - Determinação dos índices RMR por família de descontinuidade.

\begin{tabular}{|c|c|c|c|c|c|c|c|c|c|c|c|}
\hline TALUDE & FAMÍLIA & UCS & RQD & ESPAÇAMENTO & PERSISTÊNCIA & ABERTURA & RUGOSIDADE & PREENCHIMENTO & ALTERAÇão & ÁGUA & RMR \\
\hline \multirow{3}{*}{1} & $\mathrm{~F} 1$ & 15 & 20 & 8 & 2 & 0 & 5 & 6 & 3 & 15 & 74 \\
\hline & $\mathrm{F} 2$ & 15 & 20 & 15 & 2 & 0 & 5 & 6 & 3 & 15 & 81 \\
\hline & F3 & 15 & 20 & 10 & 2 & 0 & 5 & 6 & 3 & 15 & 76 \\
\hline \multirow{3}{*}{2} & $\mathrm{~F} 4$ & 15 & 17 & 10 & 2 & 1 & 5 & 6 & 3 & 15 & 74 \\
\hline & F5 & 15 & 17 & 10 & 2 & 0 & 5 & 6 & 3 & 15 & 73 \\
\hline & F6 & 15 & 17 & 10 & 2 & 0 & 1 & 6 & 3 & 15 & 69 \\
\hline \multirow[t]{2}{*}{3} & F7 & 15 & 13 & 5 & 2 & 1 & 1 & 6 & 1 & 15 & 59 \\
\hline & F8 & 15 & 13 & 8 & 2 & 0 & 1 & 6 & 1 & 15 & 61 \\
\hline \multirow{4}{*}{4} & F9 & 15 & 17 & 10 & 2 & 1 & 3 & 6 & 3 & 15 & 72 \\
\hline & F10 & 15 & 17 & 10 & 2 & 1 & 3 & 6 & 3 & 15 & 72 \\
\hline & F11 & 15 & 17 & 8 & 2 & 1 & 1 & 6 & 3 & 15 & 68 \\
\hline & F12 & 15 & 17 & 10 & 2 & 1 & 1 & 6 & 3 & 15 & 70 \\
\hline \multirow{3}{*}{5} & F13 & 15 & 17 & 10 & 4 & 1 & 5 & 6 & 3 & 15 & 76 \\
\hline & F14 & 15 & 17 & 10 & 4 & 1 & 5 & 6 & 3 & 15 & 76 \\
\hline & F15 & 15 & 17 & 10 & 4 & 1 & 5 & 6 & 3 & 15 & 76 \\
\hline \multirow{3}{*}{6} & F16 & 15 & 17 & 8 & 2 & 1 & 5 & 6 & 5 & 15 & 74 \\
\hline & F17 & 15 & 17 & 15 & 2 & 1 & 5 & 6 & 5 & 15 & 81 \\
\hline & F18 & 15 & 17 & 8 & 2 & 1 & 5 & 6 & 5 & 15 & 74 \\
\hline \multirow{3}{*}{7} & F19 & 15 & 20 & 10 & 2 & 1 & 5 & 6 & 1 & 15 & 75 \\
\hline & F20 & 15 & 20 & 10 & 2 & 1 & 3 & 6 & 1 & 15 & 73 \\
\hline & $\mathrm{F} 21$ & 15 & 20 & 10 & 2 & 1 & 3 & 6 & 1 & 15 & 73 \\
\hline \multirow[t]{2}{*}{8} & $\mathrm{~F} 22$ & 15 & 20 & 10 & 2 & 0 & 3 & 6 & 3 & 15 & 74 \\
\hline & $\mathrm{F} 23$ & 15 & 20 & 10 & 2 & 0 & 3 & 6 & 5 & 15 & 74 \\
\hline
\end{tabular}

Fonte: Autor (2021).

Em geral, para cada talude, sugere-se o estabelecimento do menor índice encontrado dentre as famílias analisadas a fim de se estar a favor da segurança. Os resultados evidenciam uma boa qualificação e competência dos taludes, com maciços rochosos variando de bom a muito bom, excetuando-se o talude 3 que tem maciço classificado como regular (Classe III).

\subsection{Determinação do SMR dos taludes}

Para o cálculo do SMR optou-se somente analisar as famílias de descontinuidades que tinham mais chances de acontecer uma ruptura planar e para isto foram utilizados os índices RMR das respectivas famílias. Consequentemente, foram analisadas as famílias 1 (Talude 1), 4 (Talude 2), 11 (Talude 4), 16 (Talude 6), 19 (Talude 7) e 23 (Talude 8); devido aos mergulhos relativos a inclinação dos seus respectivos taludes. Encontrou-se os seguintes resultados para a obtenção do índice SMR, que são apresentados na Tabela 17.

No presente trabalho, ressalta-se que o fator de ajuste F4, conforme a Equação (3), é nulo devido ao processo de corte do maciço rochoso ter sido efetivado pelo método de desmonte com explosivos e mecanicamente. Assim como, alguns valores de fatores ficam prederminados devido as conformações entre a descontinuidade e o talude. 
Tabela 17 - Valores empíricos do fator F4.

\begin{tabular}{ccccccc}
\hline $\begin{array}{c}\text { TALUDE } \\
\text { ESTUDADO }\end{array}$ & F1 & F2 & F3 & F4 & RMR & SMR \\
\hline Talude 1 & 0,15 & 0,2378 & $-29,71$ & 0 & 74 & 73 \\
Talude 2 & 0,15 & 0,3904 & $-29,57$ & 0 & 74 & 72 \\
Talude 4 & 1,00 & 0,2378 & $-29,76$ & 0 & 68 & 61 \\
Talude 6 & 1,00 & 0,15 & $-31,23$ & 0 & 74 & 69 \\
Talude 7 & 0,15 & 0,2827 & $-29,69$ & 0 & 75 & 74 \\
Talude 8 & 0,15 & 1,00 & $-32,50$ & 0 & 74 & 69 \\
\hline
\end{tabular}

Fonte: Autor (2021)

Assim sendo, com os respectivos valores de SMR para cada talude, pode-se determiner a classe do maciço de acordo com as conformidades geométricas em que ele se encontra, a Tabela 17 mostra a qualificação proposta por Romana (2003). Romana ainda determinou a qualidade da estabilidade do maciço, assim como os prováveis tipos de ruptura das encostas.

Tomando como base os valores de SMR encontrados, é fatídico que as famílias analisadas em geral encontram-se estáveis, pois ficaram categorizadas na classe II. Contudo, como previsto por Romana (2003), mínimas rupturas ainda podem ser observadas considerando a metodologia de corte e também o estado em que os taludes se encontram.

\subsection{Análise das prováveis rupturas e sugestões de melhoramento}

Esta seção destina-se aos comentários das eventuais rupturas que possam vir acontecer com o passsar do tempo, o desgaste estrutural do talude e suas possíveis metodologias de melhoramento. Embora os taludes estejam agrupados em classes estáveis, as conformações de suas descontinuidades podem promover instabilidades mínimas e locais em certas partes do talude. Isto provém do fato de o RMR classificar o maciço de forma global sem levar em conta suas conformizações geométricas localmente.

Para isto, deve-se prever estes prováveis acontecimentos e propor reforços ou alternativas de melhoramento a fim de evitar acidentes até mesmo durante o processo de corte, período ao qual acontecem as maiores incidências.

A seguir destacam-se as prováveis rupturas, para cada família de descontinuidade, de acordo com sua classificação na Tabela 18, tais rupturas são antevistas de acordo com a direção e mergulho de cada junta observados em campo.

Assim sendo, alguns taludes merecem ser destacados devido a algumas irregularidades ou peculiares configurações. O mais crítico deles, o talude 3, devido ao seu mediano RMR e sua alta densidade de juntas apresentará ocorrências frequentes de rupturas por tombamento. Contudo, seu correto distanciamento à valeta da rodovia induz suas instabilidades a serem depositadas no pé do talude sendo, portanto, uma boa prática de engenharia.

O talude 4 encontra-se com sua base desgastada, o que pode ocasionar futuras rupturas por tombamento nas partes mais superiores; adicionalmente, é visível o processo de formação de futuras rupturas planares localizadas que podem ter sido inicializadas devido a fatores não considerados neste estudo, como é o caso de sismos de magnitude pequena, ou condições de desgaste natural. Estas observações podem ser verificadas na Figura 3 nos itens de (a) à (c). Nesta mesma Figura, itens (a) e (b) o material da decomposição já inicia o processo de ocupação da valeta da rodovia, podendo futuramente com o intemperismo causar mal funcionamento do elemento projetado, e no item (c) pode-se observar um bloco já se desprendendo do maciço do talude. 
Tabela 18 - Tipo de provável ruptura observada em campo para cada família.

\begin{tabular}{|c|c|c|}
\hline TALUDE & FAMÍLIA & TIPO DE RUPTURA \\
\hline \multirow{3}{*}{1} & $\mathrm{~F} 1$ & Ruptura planar \\
\hline & $\mathrm{F} 2$ & Ruptura por tombamento \\
\hline & $\mathrm{F} 3$ & Ruptura planar/Ruptura em cunha \\
\hline \multirow{3}{*}{2} & $\mathrm{~F} 4$ & Ruptura planar \\
\hline & F5 & Ruptura por tombamento \\
\hline & F6 & Ruptura em cunha \\
\hline \multirow[t]{2}{*}{3} & F7 & Ruptura por tombamento \\
\hline & F8 & Ruptura por tombamento \\
\hline \multirow{4}{*}{4} & F9 & Ruptura por tombamento \\
\hline & F10 & Ruptura por tombamento \\
\hline & F11 & Ruptura planar \\
\hline & $\mathrm{F} 12$ & Ruptura por tombamento \\
\hline \multirow{3}{*}{5} & F13 & Ruptura por tombamento \\
\hline & $\mathrm{F} 14$ & Ruptura por tombamento \\
\hline & F15 & Ruptura planar \\
\hline \multirow{3}{*}{6} & F16 & Ruptura planar \\
\hline & F17 & Ruptura em cunha \\
\hline & F18 & Ruptura em cunha \\
\hline \multirow{3}{*}{7} & F19 & Ruptura planar \\
\hline & F20 & Ruptura por tombamento \\
\hline & $\mathrm{F} 21$ & Ruptura por tombamento \\
\hline \multirow[t]{2}{*}{8} & $\mathrm{~F} 22$ & Ruptura por tombamento \\
\hline & F23 & Ruptura planar \\
\hline
\end{tabular}

Fonte: Autor (2021).

Figura 3 - Condições de degradação e ruptura do talude 4.

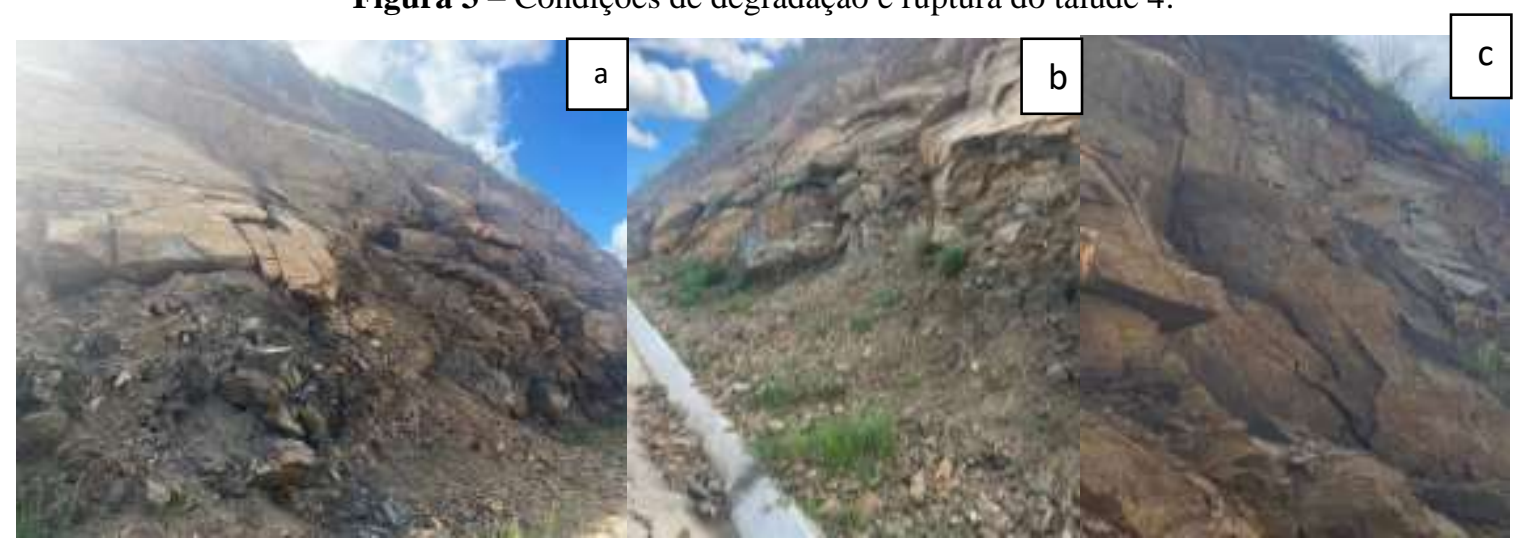

Fonte: Autor (2021).

O talude 5 apresenta espaçamento muito pequeno relativo a valeta da rodovia, em casos de ruptura por tombamento isto, por ventura, pode ser um fator inapropriado. Pode-se observar isto na Figura 4 no item (a). Notoriamente, por sua vez, o talude 6 e 7 apresentam marcas das suas anteriores rupturas por cunhas, as quais podem ser observadas na Figura 4 nos itens (b) e (c), logo demonstrando possibilidade de ocorrências de rupturas por tombamento localizadas. 
Figura 4 - Vista do espaçamento do talude 5 com a rodovia.

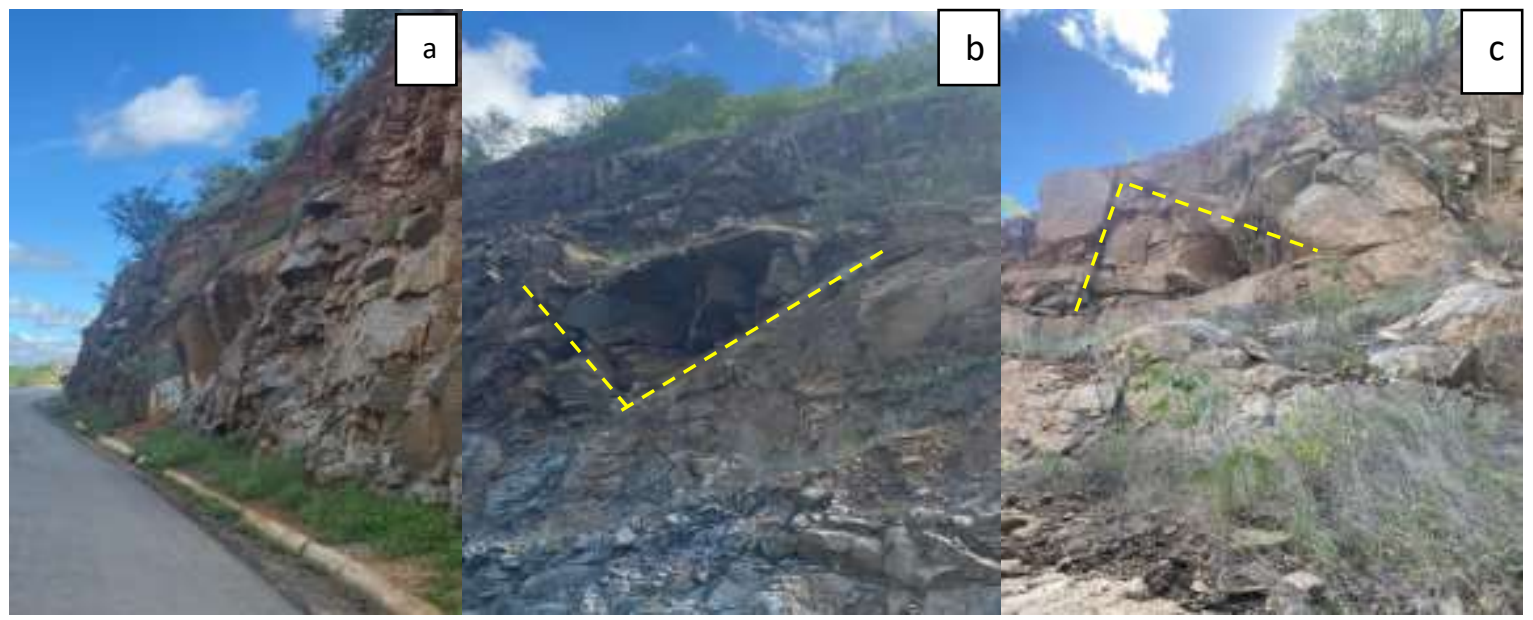

Fonte: Autor (2021).

Em resumo, constatada a estabilização dos referidos taludes e suas zonas e características de falha é possível deliberar metodologias de correção para as prováveis instabilizações dos mesmos. Romana (2003) propõe que para os taludes com SMR entre 25 e 65 aproximadamente existe a possibilidade da utilização de concreto projetado para a estabilização das pequenas quedas e para os taludes com índices variando de 45 à 75 aproximadamente possam ser aplicadas, alternativamente, telas de contenção metálicas.

No presente caso, as proposições de melhoramento concernem na retirada dos blocos grandes em contrapartida ao atirantamento e a limpeza dos blocos de menor porte nos casos em que sejam viáveis. Para o talude 4, sugere-se o rearranjo da base do mesmo e a verificação da estabilidade nas zonas onde os fenômenos de desgaste ocorrem após a sua reconformação.

\section{Conclusão}

O objetivo deste trabalho é utilizar os conceitos e metodologias de Mecânica das Rochas na intenção de caracterizar geológico-geotecnicamente os taludes rochosos originados pelo processo de duplicação de trechos da rodovia PE-160 e BR104, tal caracterização utilizou a metodologia dos índices Rock Mass Rating (RMR) e Slope Mass Rating (SMR) a fim de verificar a estabilidade dos taludes.

Analisou-se 8 taludes, os quais demonstraram condições estáveis com índices RMR de ótimos à regulares e tendências de rupturas planares globais controladas, evidenciando somente prováveis danos mínimos que são em sua maioria contidas pelas valas de drenagem ou espaçamento entre o talude e a rodovia, não causando danos consideráveis.

Por motivo de segurança, é sugerido somente a limpeza e retirada de blocos dos taludes como tática de melhoramento das condições dos taludes, uma vez que os índices definem taludes competentes.

Expressar a qualidade de um maciço rochosos em um único índice sempre tem suas dificuldades, pois de certa forma é impraticável a contagem e catalogação de todas as descontinuidades presentes no maciço, que são diversas. Neste trabalho, analisou-se somente as descontinuidades que favoreciam condições de instabilidade, por observação em campo. Contudo, há descontinuidades que quando analisadas em interação com outras podem promover certas situações de desestabilidade.

Por fim, trabalhos de análise de estabilidade de taludes como este, são fundamentais para verificar as condições estruturais de taludes, e as práticas executivas em obras lineares para cortes em maciços. Portanto, certificando a segurança de estruturas como estas ou antevendo ocorrências danosas e minimizando os riscos à sociedade. 
Research, Society and Development, v. 10, n. 16, e209101623637, 2021

(CC BY 4.0) | ISSN 2525-3409 | DOI: http://dx.doi.org/10.33448/rsd-v10i4.23637

Além de, academicamente, contribuir para a difusão dos conhecimentos de Mecânica das Rochas pouco conhecidos por estudantes de engenharia, bem como, socialmente, apresentando seguridade, melhoramentos alternativos para obras civis como esta e ajudando em futuras tomadas de decisões. Por fim, para trabalhos futuros fica sugestivo a aplicação dos dados obtidos neste trabalho para outras metodologias de classificação geomecânica e determinação demais índices relacionados a temática como bem visto em Santos et al. (2020).

\section{Referências}

Barton, N. (1974). Engineering Classification of Rock Masses for the Design of Tunnel Support. Rock Machanics and Rock Engineering, 6, p. $189-236$.

Barton, N., Choubey, V. (1977). The shear strengh of rock joints in theory and practice. Rock Mechanics. Vienna: Springer. p 1-54.

Bieniawski, Z. T. (1973). Engineering classification of jointed rock masses. Transactions of South Africa Institute of Civil Engineers. 15, p. $344-355$.

Bieniawski, Z. T. (1989). Engineering rock mass classifications. New York, John Wiley. 251p.

CPRM (Brazilian Geological Survey). (2017). Geologia e recursos minerais da folha Santa Cruz do Capibaribe - SB.24-Z-D-VI. Recife.

CPRM (Brazilian Geological Survey). DNPM (National Department of Mineral Production). (2001). Geological Map of Pernambuco State. Scale 1:100.000.

Deere, D. U.; Hendron, A. J.; Patton, F. D.; Cording, E. J. (1967). Design of surface and near-surface construction in rock. Symposium on Rock Mechanics American Institute Mining, Metallurgical and Petroleum Engineers. Mineapolis. p. 237-302.

Goodman, R. E. (1989). Introduction to rock mechanics. 2nd ed. Willey. New York. 562p.

Hoek, E. (1983). Strength of Jointed Rock Masses. Geotechnique, 33 (3), p.187-223.

Hoek, E., Brown, E. T. (1997). Practical estimates of rock mass strength. International Journal of Rock Machanics and Mining Science and Geomechanics Abstracts. 27 (3). p. 227-229.

Hoek, E., Kaiser, P. K. and Bawden, W. F. (1995). Support of underground Excavation in Hard Rock. Rotterdam: Balkema. p. 215.

ISRM. (1981). Basic geotechnical description of rock masses. International Journal of Rock Mechanics and Mining Sciences \& Geomechanics Abstracts. 18, p. $85-110$.

ISRM. (1981). Suggested Methods for the Quantitative Description od Discontinuities in Rock Masses. International Journal of Rock Mechanics and Mining Sciences \& geomechanics Abstracts. 15, p. 319-368. 1981.

Lima Neto, H. C. (2013). Sismicidade e correlação com feições geoloógicas: o caso do Lineamento Pernambuco e seu entorno. Tese de Doutorado, $P P G G / U F R N$. Natal. 171p.

Palmstrom, A. (1996). Characterizing rock masses by the RMi for Use in Practical Rock Engineering. Tunn. Undergr. Spec. Tech. 11 (2), p. $176-188$.

Palmstrom, A. (2000). Measurement and Characterization of Rock Mass Jointing. In-situ characterization of rocks. Balkema Publishers. Oslo. p. 49-97.

Palmstrom, A. (2005). Measurements of and correlations between block size and rock quality designation (RQD). Tunneling and Underground Space Technology. 20, p. 362-377.

Romana, M. (1993). A geomechanics classification for slopes: slope mass rating. Comprehensive Rock Engineering. London: Pergamon. p. 575-600.

Romana, M. (1985). New adjustment ratings for application of Bieniawski classification to slopes. International Symposium on the Role of Rock Mechanics ISRM. Zacatecas. p. 49-53.

Romana, M.; Serón, J. B.; Montalar, E. (2003). SMR geomechanics classification: application, experience and validation. Journal Nielen van der Merwe. 10th Congress of the International Society for Rock Mechanics. Saldton. p. 8-12.

Santos, A. E. M.; Silva, D. de F. S. da; Mendonça, G. A.; Santos, T. V.; Amaral, R. R.; Silva, L. A. M. (2020). Rockfall index and geological-geotechnical characterization on road slopes: a case study on BR-262. Research, Society and Development, [S. l.], 9, n. 12, p. e12891210968.

Tabalipa, N. L. (2008). Estudo da estabilidade de vertentes da bacia do Rio Ligeiro, Pato Branco, Paraná. Tese de doutorado, PPGEOL/UFPR. Curitiba. 243p.

Tonus, B. P. A. (2009). Estabilidade de taludes: Avaliação dos métodos de equilíbrio limite aplicados a uma encosta coluvionar e residual da serra do mar paraense. Tese de mestrado, PPGEC/UFPR. Curitiba. 147p. 